\title{
L'usage, fondement de la sentence rendue par le juge administratif camerounais
}

\author{
Par François-Narcisse Djame, Douala
}

\section{Introduction}

L'étude des usages dans la jurisprudence administrative peut paraitre vaine ou, tout simplement, se révéler hasardeuse. Essentiellement juge du droit écrit, le juge administratif a pour mission première d'appliquer la loi (lato sensu) dans le but de départager les parties qui se soumettent ou sollicitent sa sentence.

La mission d'application de la loi dévolue au juge est beaucoup plus marquée, voire justifiée, dans les systèmes juridiques qui rejettent le droit coutumier en général, au regard de la place axiale que ces systèmes accordent au principe de la souveraineté nationale ${ }^{1}$. Suivant ce principe, ainsi que le soulignait Esmein, «le droit national et la loi se confondent ${ }^{2}$. On comprend pourquoi le droit que le juge administratif se charge d'appliquer est celui fixé par la loi, suivant la logique «normativiste ${ }^{3}$. Dans cette optique, la reconnaissance du droit

1 L'art. 2(1) de la Constitution du 02 juin 1972, révisée par la loi du 18 janvier 1996, dispose que « la souveraineté appartient au peuple camerounais qui l'exerce soit par l'intermédiaire du Président de la République et des membres du Parlement, soit par voie de référendum. Aucune fraction du peuple ni aucun individu ne peut s'en attribuer l'exercice ».

La position que H. Capitant adopte en faveur de la coutume malgré le principe de la souveraineté nationale semble isolée. L'auteur soutient, en effet, qu'en déléguant à des représentants le droit de faire la loi, la nation n'a pas entendu tarir la source coutumière, distincte de la loi. Voir Introduction au droit civil, p. 47, cité par A. Lebrun, La coutume, ses sources, son autorité en droit privé, thèse, Paris, LGDJ, 1932, p. 394.

A. Esmein, «La coutume doit-elle être reconnue comme source du droit civil français ? », Rapport présenté à la première Sous-commission de révision du Code civil, Bulletin de la Société d'Etudes Législatives, Paris, Arthur Rousseau, 1905, p. 4.

3 Carl Schmitt oppose le décisionnisme des Anglo-Saxons suivant lequel le droit est fixé progressivement par le juge au regard des précédents judiciaires, au normativisme des systèmes juridiques continentaux européens où le droit est surtout fixé par les normes législatives préétablies. Voir, Paul Roubier, «L'ordre juridique et la théorie des sources du droit », Mél. Ripert, Paris, LGDJ, 1950, p. 14 
coutumier comme caractéristique du pluralisme juridique camerounais, peut paraître contraire à la Constitution ${ }^{4}$.

En marge des considérations d'ordre constitutionnel et purement théoriques, les principales tares que l'on attribue généralement au droit coutumier et qui constituent des obstacles pratiques à sa connaissance, sont l'incertitude et l'imprécision ${ }^{5}$. Dès lors, il n'est pas sans intérêt de se demander par quel procédé le juge administratif parvient à explorer l'univers sinueux et mystérieux de l'usage, en particulier. Plus précisément, comment le juge se réfère-t-il aux usages pour dire le droit en droit administratif camerounais ?

Les juristes camerounais se sont tenus à ce jour à l'écart de cette préoccupation ; et leur attitude semble se justifier. Habitués à un juge soucieux de protéger la légalité formelle ${ }^{6}$, ces auteurs n'imaginent peut-être pas, ou n'envisagent pas que le juge administratif puisse appliquer autre chose que la loi (et les principes généraux du droit). C'est ce qui pourrait expliquer qu'aucune étude n'ait été consacrée à ce jour aux usages en matière administrative en droit camerounais ${ }^{7}$. Pourtant, il est avéré que, dès l'aube de son office au Cameroun

Le droit coutumier visé ici est la coutume, au sens de règle de droit; il n'est pas synonyme de «droit traditionnel» reconnu en droit privé camerounais. S'il est admis que le juge camerounais applique la coutume au même titre que la loi et le règlement ( en vertu de l'article 23 du décret $\mathrm{n}^{\circ} 95 / 048$ du 8 mars 1995 portant statut de la magistrature, relatif au serment de magistrat, ce dernier s'engage lors de sa prestation de serment dès son entrée dans la magistrature, «à rendre justice avec impartialité, conformément aux lois, règlements et coutumes du peuple camerounais »), cette reconnaissance de la coutume dans l'ordre juridique camerounais ne semble pas conforme à la Constitution.

Par ailleurs, affirmer que la coutume a été érigée au rang de règle de valeur constitutionnelle semble excessif, comme l'écrit M. Célestin Sietchoua Djuitchoko (Lire «Du nouveau pour la coutume en droit positif camerounais: la constitutionnalisation de la coutume et ses conséquences »), Revue Juridique Thémis (Canada) n ${ }^{\circ} 1$, 2000, p. 131-157. Pour cet auteur, le fait que la Constitution en son article 1(2) dispose que la République du Cameroun « reconnaît et protège les valeurs traditionnelles conformes aux principes démocratiques, aux droits de l'homme et à la loi », équivaut à une reconnaissance de la valeur constitutionnelle à la coutume par la loi fondamentale. On note que M. C. Sietchoua établit une synonymie entre la coutume, règle de droit, et les «valeurs traditionnelles ». Cette conception extensive de la notion de coutume nous parait inacceptable.

A. Esmein, « La coutume doit-elle être reconnue... », op. cit., p. 9.

6 Cette attitude est clairement exprimée par le juge administratif dans le jugement Sté Assureurs Franco-Africains (n ${ }^{\circ} 62 / \mathrm{CS} / \mathrm{CA}$ du 25 sept. 1980), infra, note 51.

Exception faite de la récente thèse de doctorat de l'auteur portant sur la «contribution à l'étude des usages et de la coutume en droit administratif camerounais », thèse, Paris XII, 31 mars 2004. 
et jusqu'à ce jour ${ }^{8}$, le juge administratif s'intéresse aux usages, qu'il applique de façon autonome, indépendamment de la loi ${ }^{9}$.

L'intervention du juge administratif dans le domaine mouvant des usages semble trouver, dans l'ensemble, une justification dans l'universalité de la pensée inaltérable de Portalis, exprimée dans le «Discours préliminaire » au projet de Code civil français, en ces termes : «A défaut d'un texte précis sur chaque matière, un usage constant et bien établi... tient lieu de loi ${ }^{10}$. Cette préoccupation de Portalis rejoint la pensée de Gény, dont les travaux sur la renaissance du droit coutumier ont révélé la place de la coutume comme source du droit, et par là, l'incapacité de la loi à régir tous les rapports juridiques ${ }^{11}$.

Contrairement au juge judiciaire camerounais qui applique directement la «coutume » comme règle de droit ${ }^{12}$ dans le cadre des tribunaux de droit traditionnel notamment, la

L'histoire de la juridiction administrative au Cameroun s'adapte à l'évolution politique du pays. Quatre étapes ont ponctué cette évolution : le Conseil du Contentieux Administratif (1920-1959) ; le Tribunal d'Etat (1959-1961) ; la Cour Fédérale de Justice (1961-1972), et, depuis 1972, la Cour Suprême, avec une Chambre Administrative et une Assemblée Plénière, statuant en matière administrative, respectivement en premier ressort et en appel.

Cette étude ne prend pas en compte les usages «confirmés par la loi », i.e., ceux auxquels les textes renvoient. L'étude de ces usages que l'on rencontre essentiellement dans la jurisprudence des chefferies traditionnelles ne semble pas d'une grande utilité, du moins, dans ce cadre. En effet, lorsque le juge administratif saisi d'un recours pour excès de pouvoir sanctionne la violation des usages par l'autorité administrative, il le fait indirectement, par l'intermédiaire de la loi, étant donné qu'il annule l'acte administratif contesté pour violation de la loi ( art. 11 du décret de 1977 portant organisation des chefferies traditionnelles) et non pour violation de la «coutume». Le jugement Collectivité Deïdo de Douala ( $\mathrm{n}^{\circ} 63 / \mathrm{CS} / \mathrm{CA}$ du 25 sept. 1980) est significatif à cet égard. Le juge décide "qu'en procédant à la consultation des chefs de quartier au détriment des chefs des familles du canton Deïdo (ainsi que l'exige la « coutume »), l'administration a violé les dispositions de l'article 11...». Voir l'opinion contraire exprimée par C. Sietchoua DJ., «Aspects de l'évolution des coutumes ancestrales dans le droit public des chefferies traditionnelles », Revue Générale de Droit de l'Université d'Ottawa, n³2, 2002, p. 375.

10

Fenet, t. 1, p. 469, cité par Louis Bach, Droit civil, tome I, 13è éd., Dalloz 1999, p. 56, note 98.

11 F. Geny, Méthode d'interprétation et sources en droit privé positif, tome I, Paris, LGDJ, 1954, p. 349.

12

Les études révélant que la coutume est règle et source du droit au Cameroun ont surtout été menées par les privatistes. Parmi les plus récentes, on peut citer l'intéressant article du Professeur Victor-Emmanuel Bokally, intitulé « La coutume, source de droit au Cameroun », Revue Générale de Droit de l'Université d'Ottawa, n 28, 1997, p. 37-69. Cet intitulé peut paraitre trompeur. On peut en effet penser que cette étude concerne le droit coutumier dans son ensemble, pourtant, la lecture de l'article traduit le sentiment communément partagé qu'il n'y a coutume qu'en droit privé au Cameroun.

Il faut néanmoins signaler la place de la «coutume» en droit administratif dans les travaux de deux publicistes camerounais, portant sur le droit des chefferies traditionnelles. Il s'agit de RogerGabriel Nlep, L'administration publique camerounaise. Contribution à l'étude des systèmes afri- 
méthode de connaissance du droit coutumier par le juge administratif présente la particularité de partir de l'observation des usages, «matière première » ${ }^{13}$ de la coutume. S'il arrive, dans certains cas, au juge de révéler une règle coutumière qui se forme au sein des groupes sociaux tels que les localités, les administrations ou les entreprises, cette opération de «mise au jour » de la coutume découle implicitement du caractère prescriptif ou permissif de l'usage ${ }^{14}$.

Les développements qui suivent ne se limitent pas à rechercher la normativité des usages appliqués par le juge administratif camerounais. Une telle approche ne manque pourtant pas d'intérêt, dans la mesure où elle oriente l'analyse dans l'étude de la question théorique de la «transmutation du fait en droit » ${ }^{15}$, qui se situe sans doute au cœur de l'étude du droit coutumier ${ }^{16}$. Toutefois, cette démarche présente l'inconvénient de réduire considérablement le champ d'intervention des usages dans la jurisprudence, et altère la richesse qu'offre leur observation, à travers leur diversité. Cette étude prend plutôt appui sur la connaissance des usages, comme faits indispensables à la résolution de certains litiges soumis à l'appréciation du juge administratif (I). En effet, si l'usage peut «tenir lieu de loi » dans une situation donnée, cela ne veut pas dire qu'il change automatiquement de nature et devient règle de droit. Tout en demeurant au stade du simple fait, l'usage peut jouer le rôle qui est ou qui serait attendu d'une règle de droit écrit dans une matière donnée $^{17}$. La certitude de l'usage constitue par conséquent le préalable à l'action du juge

cains d'administration publique, Paris, LGDJ, 1986, p. 127 s., et Célestin Sietchoua DJ., «Aspects de 1'évolution des coutumes ancestrales... », préc., note 9, in fine.

13

14

La doctrine admet généralement que le juge énonce l'usage sous une forme normative, en soulignant qu'en vertu de l'usage, tel comportement doit avoir lieu (norme prescriptive), ou peut avoir lieu (norme permissive). Voir cette distinction, notamment, chez Hans Kelsen, Théorie Pure du Droit, trad. Ch. Eisenmann, LGDJ, Paris, 1999, p. 13 ; Théorie générale des normes, PUF, Paris, 1996, p. 3 ; et, pour ce qui est spécifiquement du droit administratif, dans l'ouvrage du Professeur Gérard Teboul, Usages et coutume dans la jurisprudence administrative, Paris, LGDJ, 1989, Deuxième et Troisième Parties, p. 102-295.

M. Troper, « Du fondement de la coutume à la coutume comme fondement », Droits, $\mathrm{n}^{\circ} 3,1986$, p. 12.

16 Le Professeur Gérard Teboul note que la distinction entre le fait et le droit constitue «le mystère sous-jacent à toute l'étude du droit coutumier ». lire Usages et coutume..., préc., p. 103.

17

Dans un arrêt Commune de Port-Marly (C.E., 16 nov. 1984, décision non publiée), la Haute Juridiction administrative française s'est appuyée sur un usage pour déterminer le tracé existant entre deux communes, sans consacrer une règle coutumière. Le Conseil d'Etat saisi en appel, a jugé «qu'en l'absence d'autres titres suffisamment précis, c'est à bon droit que le tribunal administratif s'est référé aux usages et coutumes pour en arrêter le tracé ». Voir Gérard Teboul, Usages et coutume..., p. 122, note 39 
administratif et justifie son intervention lorsqu'il sollicite l'usage ou l'applique dans certains litiges (II).

\section{La certitude de l'usage, condition de son application par le juge administratif}

L'usage se décline au juge administratif camerounais de diverses façons. Le juge dévoile directement l'usage dont il a connaissance au cours de l'instruction (A); tout comme, de son propre chef, il s'efforce de rechercher l'usage invoqué ou non par une partie. Dans ce cas, il recourt à certaines techniques que lui offrent les mesures d'instruction, et qui constituent autant d'éléments de nature à révéler l'existence de l'usage (B).

\section{A. L'observation directe de l'usage}

Le juge administratif adopte une démarche particulière lorsqu'il se prononce sur l'existence de l'usage. Contrairement aux autres faits portés à sa connaissance, le juge fait sienne une certaine attitude qui confirme que l'existence d'une pratique alléguée dans un litige ne fait pas de doute. Par ailleurs, lorsque le juge n'a pas directement la certitude de l'usage, il recourt à un complément d'information dans le but d'asseoir sa conviction.

\section{La référence aux caractères de l'usage comme critères de sa connaissance}

Lorsque le juge administratif admet l'existence de l'usage, il emprunte deux directions qui, en fait, se ramènent à une seule. Il peut arriver, premièrement, que le juge révèle l'usage au travers de ses caractères. C'est le cas de la pratique «constante » ${ }^{18}$ qu'il signale dans certaines de ses décisions. Il asseoit par là le caractère irréfragable de l'usage. En effet, quand le juge administratif révèle une pratique constante, cela signifie qu'il est convaincu de l'existence de la pratique ${ }^{19}$ concernée.

Dans l'arrêt Emougou Michel-Gabriel ( ${ }^{\circ}{ }^{\circ}$ 18/CS/AP, 5 juillet 1973), le juge administratif décide «...qu'il est de pratique constante que le Premier Ministre nomme par arrêté les cadres subalternes de la Sûreté nationale ».

19

La terminologie variée renvoyant à l'usage ne constitue pas, a priori, un obstacle à la compréhension du phénomène de l'usage. A notre sens, il faut s'attacher au contenu de l'usage (i.e. un comportement réitéré observé au sein d'un groupe social, en dehors des textes) et non à sa dénomination. Ainsi, les termes, «pratique », « habitude», «coutume administrative », «tradition », « coutume », etc., rencontrés dans la jurisprudence, sont synonymes et renvoient à l'usage. Il est par ailleurs significatif de noter que le juge administratif camerounais n'utilise rarement le mot « usage » dans ses décisions que pour rappeler les prétentions des parties. On observe cette atti- 
On note également, en deuxième lieu, que lorsque le juge administratif se réfère à un usage sans dire que celui-ci est «constant», ou qu'une pratique est «courante » ${ }^{20}$, il reconnaît, implicitement, le caractère de constance de l'usage considéré. On observe cette attitude du juge dans le jugement Ngoumou Richard ${ }^{21}$, dans lequel la Chambre Administrative de la Cour Suprême se contente de solliciter une «pratique », sans l'assortir formellement d'un qualificatif quelconque renvoyant à l'un de ses caractères. Aussi, lorsque le juge constate l'existence d'une pratique au cours de l'instruction d'une affaire, et qu'il révèle l'usage en cause, il faut nécessairement admettre qu'il intègre la constance de la pratique dans son raisonnement. Il peut donc paraître tautologique d'exiger d'une coutume administrative, par exemple, qu'elle soit « constante ». Dès lors que cette « coutume» est sollicitée par le juge, celui-ci atteste de la certitude de l'usage, et sa constance ne fait plus de doute ${ }^{22}$.

L'attitude du juge administratif permet par conséquent de relever qu'il est attaché aux caractères de l'usage et notamment à sa constance ${ }^{23}$. On peut donc dire que la constance caractérise tout usage. Il est dès lors permis de noter que le juge exige, sans doute, au cours de l'instruction de l'affaire, que les faits portés à sa connaissance soient constants. Mais il demeure libre de ne pas faire ressortir les caractères de l'usage dans les motifs de sa décision ; ce qui importe, c'est l'existence de l'usage qu'il confirme.

Il faut signaler toutefois que la démarche du juge administratif camerounais est particulière, relativement au caractère d'ancienneté de l'usage qui, avec la constance, constitue les caractères «intrinsèques » de l'usage ${ }^{24}$. En effet, les expressions «usages anciens » ou

tude dans l'arrêt Njikiakam Towa Maurice ( CS/AP, 24 mars 1983), où le juge relève «que par ailleurs, compte tenu des usages, le requérant avait à rejoindre son nouveau poste dans les quinze jours suivant la notification de l'acte d'affectation ».

CCA, décision ${ }^{\circ} 16$ du 7 septembre 1945, Esse Stanislas c/ Administration du Territoire.

21 CS/CA, n ${ }^{\circ} 17$ du 23 novembre 1989.

22

De façon éclatante, le juge administratif affirme l'existence de la « coutume administrative » dans l'arrêt Dame Mackongo Agnès-Flore (n'201/CFJ/CAY, 18 août 1972) Cf. infra, note 29 et Ilème Partie de l'étude, note 45.

23 Si l'attachement du juge à l'usage se révèle en général à travers ses caractères formels (constance, ancienneté), il s'appuie, certes rarement, sur ses caractères matériels. On comprend pourquoi le juge administratif camerounais n'hésite pas à rejeter une coutume administrative invoquée par un requérant dans le paiement des heures supplémentaires de travail, parce qu'elle manque de fixité ( ${ }^{\circ}$ 4/CCA, 7 fév. 1938, Pignon). Mais on peut penser, dans cette affaire, que la fixité est un caractère constitutif de l'usage. En réalité, la fixité intègre sa constance, puisque c'est le paiement des heures supplémentaires de travail, i.e. l'usage, qui est constant, et non le taux en usage qui lui, manque de fixité. pratique individuelle, même constante, n'accède pas à la qualité de l'usage. Lire Mme Pascale Deumier, Répertoire de Droit Civil, t. IV « coutume et usages », Dalloz 2003, p. 4, § 16. 
«pratiques anciennes », sont étrangères au langage du juge. Nulle part, on ne voit le juge administratif se référer à l'ancienneté pour fonder l'existence de l'usage. Cette attitude peut s'expliquer sans doute par le fait que le juge administratif estime qu'une pratique constante est dotée d'un minimum d'ancienneté. Dans ce cas, parler d'un usage « ancien et constant » peut paraître redondant. Cette vision des choses semble cohérente, car l'ancienneté de l'usage est d'une appréciation relative, tandis que sa constance, c'est-à-dire, la régularité de la pratique, est un caractère obligatoire de l'usage, et permet d'asseoir sa certitude ${ }^{25}$. Aussi, quand le juge se réfère à un usage, il a la certitude de son caractère ancien, car la constance de l'usage intègre son ancienneté ${ }^{26}$.

Tout en admettant cette réalité, il ne semble pas superflu de lier la jeunesse de l'administration camerounaise et son corollaire, le droit administratif, à l'absence presque générale des décisions se fondant sur l'«ancienneté » des pratiques administratives en particulier $^{27}$. Cette vision des choses semble être en harmonie avec la position de la doctrine qui fonde l'inexistence de la «coutume administrative » sur la fragilité des pratiques, liée à la jeunesse du droit administratif camerounais ${ }^{28}$. Mais l'ancienneté de ces pratiques, sans être formellement invoquée, ne fait pas de doute, à partir du moment où les pratiques administratives trouvent une place dans la jurisprudence du juge administratif ${ }^{29}$.

\section{L'examen des documents probants}

Le juge administratif joue un rôle déterminant dans la preuve de l'usage. Comme le conseille Gény, la preuve du droit coutumier est une question pratique qu'il convient

Le Professeur Gérard Teboul relève à ce sujet, qu' "il est clair que c'est une obligation pour l'usage d'être constant», Usages et coutume..., p. 41.

26

Les Professeurs Auby et Plaisant notent que l'ancienneté de l'usage et son caractère «courant », font partie intégrante de sa constance, in Le droit des appellations d'origine, Paris, Librairies Techniques, 1974, p. 62, n²138, cité par G. Teboul, Usages et coutume..., p. 43, note 62.

27

Le caractère d'ancienneté de l'usage a néanmoins été porté à l'appréciation du juge administratif camerounais. Dans le jugement Itondo Toko Blaise ( ${ }^{\circ} 126 / C S / C A, 25$ juillet 1991), par exemple, le requérant fondait sa revendication sur des pratiques anciennes suivies dans le paiement des heures supplémentaires de travail au port de Douala, «avant l'indépendance du Cameroun ». Malheureusement, le juge administratif a annulé l'arrêté de révocation de l'intéressé, non parce qu'il n'a pu établir l'ancienneté de l'usage allégué, mais parce que ce texte a retenu à son encontre la « concussion », constitutive d'une infraction pénale.

En reconnaissant que le jeune droit administratif camerounais n'a pas encore donné lieu à des pratiques «passées » dans la coutume administrative (Droit administratif spécial, Cours polycopié, Université de Yaoundé, 1989, p. 20). Norbert Nkameni établit, implicitement, un lien entre la jeunesse de l'administration et l'ancienneté des pratiques administratives.

Cf. arrêt Dame Mackongo Agnès-Flore, supra, note 22. 
d'abandonner au juge ${ }^{30}$. Le juge camerounais adopte une attitude de liberté quand il recherche l'usage. Il procède à une adaptation des techniques de preuve au phénomène mouvant et incertain que constitue l'usage. Quant à la question de savoir comment l'autorité juridictionnelle révèle l'usage, on note que, lorsque le juge administratif observe directement l'usage, il procède à un examen de certains écrits ou documents qui fondent sa conviction.

La certitude de l'usage peut être établie devant le juge à travers l'examen des documents qui lui sont présentés au cours de l'instruction de l'affaire, et qui préexistent au litige. C'est ce que révèle l'arrêt Ndjeudji Maurice ${ }^{31}$, relatif à la promotion des magistrats. L'Assemblée Plénière de la Cour Suprême fait droit au requérant en jugeant que, «considérant que ces documents font de même apparaître que le cumul de grade et de franchissement d'échelon à la même date, était érigé en règle... ». On objectera que l'attitude du juge administratif ne révèle pas, dès l'abord, l'usage. Il ne dit pas, par exemple, qu'une « pratique » est établie dans le groupe social concerné. Mais il ne fait point de doute que la règle, constituée par une suite de précédents, consacre l'usage, clairement établi dans le raisonnement du juge.

Ce mode de preuve n'est pas dénué d'efficacité. Non seulement il est rapide, parce qu'il permet au juge administratif de trancher de façon diligente le litige, il asseoit une fois pour toutes la conviction du juge, qui n'a plus de doute sur l'existence de l'usage, et par conséquent ne recourt plus à d'autres modes de preuve, comme il le fait dans d'autres circonstances.

\section{B. La preuve indirecte de l'usage}

La doctrine enseigne que lorsque le juge n'a pas une connaissance suffisante de l'usage, il peut prendre l'initiative d'en requérir la preuve, soit d'office, soit auprès de la partie qui $l^{\prime}$ invoque ${ }^{32}$. La jurisprudence administrative camerounaise révèle que le juge recourt à l'enquête pour établir la preuve de l'usage, lorsqu'il n'est pas certain de son existence. C'est cette dernière attitude qui, de façon isolée, mais non moins significative, est observée dans l'affaire Menyé Christophe ${ }^{33}$.

30 F. Geny, Méthode d'interprétation et sources en droit privé positif, $2^{\text {ème }}$ édition, Paris, LGDJ, 1954, p. 351.

31 CS/AP, $n^{\circ} 4$ du 26 octobre 1978.

32 F. Geny, Méthode d'interprétation..., op. cit., p. 354.

33 Cette décision a été rendue en deux étapes : le premier jugement (avant dire droit), nº63/CCA, du 5 juillet 1957 ; et celui du fond, nº51/CCA, du 25 octobre 1957. 
L'enquête ordonnée par le juge administratif est particulière; elle traduit la liberté d'action du juge dans la recherche de l'usage. Contrairement à l'enquête prévue dans la procédure administrative contentieuse ${ }^{34}$ qui se limite au seul témoignage quand le juge administratif recherche la preuve des autres faits ${ }^{35}$, l'enquête en matière d'usages associe le témoignage aux documents écrits ${ }^{36}$. Mais cela ne signifie pas que l'autorité juridictionnelle soit liée par cette attitude ; d'ailleurs, dans la présente enquête, les écrits devraient être consultés «le cas échéant », c'est-à-dire, éventuellement.

On peut penser que cette orientation de l'enquête n'est pas gratuite; le juge accorde sans doute plus d'importance à la preuve testimoniale qu'aux écrits, quand il procède à une enquête dans la recherche de l'usage. Cette démarche lui semble dictée par sa conscience, et paraît plus efficiente dans la recherche de l'usage. L'attitude du juge administratif rejoint ainsi l'opinion du Professeur Teboul, qui estime qu'en matière d'usage, «non seulement l'écrit n'est pas supérieur au non écrit, mais encore que le non écrit est supérieur à l'écrit ${ }^{37}$. Cette idée «révolutionnaire », qui ne semble pas s'éloigner de l'hérésie peut «bousculer» bien de préjugés ${ }^{38}$ dans la pensée juridique traditionnelle. Elle trouve une justification dans le fait que l'écrit présente l'inconvénient de ne donner la photographie de l'usage qu'au moment où le document est établi ${ }^{39}$. Or l'usage, par sa nature mouvante, est

Il n'a pas été possible d'avoir accès aux textes organisant la procédure administrative contentieuse à l'époque de l'affaire Menyé. Il est néanmoins donné de penser que l'enquête pratiquée de nos jours s'inscrit dans la continuité des textes coloniaux qui limitaient sans doute l'enquête au seul témoignage verbal. Pour le texte en vigueur, voir loi nº75/17 du 8 décembre 1975 fixant la procédure devant la Cour Suprême statuant en matière administrative, Chap. II, « Des mesures d'instruction ».

Voir loi $\mathrm{n}^{\circ} 75 / 17$ susvisée.

Dans le but de s'enquérir de l'existence de la «coutume » dans la désignation du chef supérieur des Ntoumou que conteste le sieur Menyé, le Conseil du Contentieux Administratif a commis le président du tribunal d'Ebolowa, lui demandant «de se faire remettre le cas échéant par l'autorité qui les détient, les accusés de réception, les listes d'émargement ou cahiers de transmission de convocations, et en procédant à toutes auditions utiles des témoins... ».

G. Teboul, Usages et coutume..., p. 96.

On admet généralement, en droit français notamment, que l'écrit est supérieur au non écrit, malgré l'antériorité de ce dernier. La règle «témoins passent lettres » a vite cédé devant sa rivale, « lettres passent témoins ». Cette question s'est posée dans l'histoire du droit. Elle consistait à savoir si l'écrit était l'expression d'une pensée plus sûre que la preuve par témoignage, par rapport à la réalité. Ch. Perelman situe ce débat par rapport à l'histoire de l'alphabétisation. Il note, en effet, qu' «on conçoit que, dans un siècle d'analphabétisme, on admette la règle « témoins passent lettres » et que dans un autre où l'instruction est répandue, on applique la règle opposée « lettres passent témoins ». Lire, La spécificité de la preuve juridique, t. 1, 1959, p. 663, cité par $P$. Foriers, «Introduction au droit de la preuve », Travaux du Centre National de Recherches de Logique, Etablissements E. Bruylant, Bruxelles, 1981, p. 19.

G. Teboul, Usages et coutume..., p. 96. 
susceptible d'évoluer de la date de sa consignation dans un document à celle de survenance du litige. Il faut donc, souligne toujours le Professeur Teboul et sauf à dénaturer l'idée même de justice, sacrifier l'efficacité, c'est-à-dire la rapidité dans la résolution du litige que garantit le document écrit, pour s'attacher à la recherche de la véritét ${ }^{40}$, que le témoignage est plus à même de fournir.

L'usage en cause dans la jurisprudence du juge administratif camerounais n'est pas «consigné » dans un écrit pour présenter les inconvénients signalés en droit français ${ }^{41}$. Ainsi par exemple, le rapprochement des documents écrits sollicités par le juge présente l'avantage de révéler l'évolution de la pratique en matière de représentation des chefs traditionnels à la cérémonie de désignation d'un chef supérieur dans la localité concernée. En effet, parce qu'ils contiennent des informations relatives à cette pratique sur plusieurs années, ces documents rendent plus compte de l'évolution de l'usage, que n'établit un recueil d'usages. Malgré cet avantage réel, le témoignage s'avère plus crédible aux yeux du juge administratif parce qu'il donne la meilleure représentation de l'usage au moment du litige.

Si le juge recourt également aux écrits, même à titre facultatif, c'est sans doute parce qu'il estime que cette approche complémentaire lui donne la meilleure connaissance de l'usage. Mais c'est le témoignage qui paraît déterminant dans la preuve de l'usage en matière d'enquête. Par rapport à la situation présentée dans l'affaire Ndjeudji où les documents pouvaient entraîner directement l'adhésion du juge parce qu'ils sont officiels, en revanche, ce dernier semble s'entourer d'autres garanties lorsqu'il est confronté à de «simples » écrits $^{42}$. Par ailleurs il demeure libre de restreindre l'enquête au seul témoignage ou à l'examen des documents écrits. Si tout est fonction de la nature du litige, le principe de liberté qui guide l'action du juge administratif l'autorise à exploiter toutes les voies possibles, en vue de la manifestation de la vérité.

La rédaction de l'usage ne tient pas souvent compte de la nature de l'usage, qui est toujours susceptible d'évoluer. Sur le plan purement technique, cette entreprise n'est pas aisée, et pas toujours fiable. Le Professeur Teboul révèle que les juristes compétents qui procèdent à la rédaction de l'usage, étrangers au groupe social où l'usage s'est formé ont de la peine à le comprendre ou à l'interpréter. De même les praticiens qui se livrent à la rédaction de l'usage et dont la formation juridique n'est pas toujours suffisante, éprouvent des difficultés à formuler abstraitement une règle. Lire Usages et coutume..., p. 96.

Les listes d'émargement, les cahiers de transmission des convocations, et les accusés de réception exigés par le juge administratif dans l'affaire Menyé, s'ils ont une certaine importance, ils ne peuvent avoir la même force probante que les actes administratifs signalés dans l'arrêt Ndjeudji, supra, note 31 . 
Mais les différents documents, et surtout les témoignages, demeurent soumis à l'appréciation du juge administratif à l'issue de l'enquête, à travers le procès-verbal. C'est en cela que l'enquête, comme mode de preuve de l'usage, est qualifiée d'indirecte. Le juge reste tout aussi libre de prescrire une autre enquête, s'il n'est pas convaincu de l'existence de l'usage révélé par le procès-verbal de la première enquête. La recherche de la vérité en dépend.

La liberté d'action du juge administratif est donc effective au regard de la complexité de la matière de l'usage. On comprend pourquoi il n'hésite pas à appliquer la pratique qui se présente à lui avec certitude.

\section{L'application de l'usage par le juge administratif et ses manifestations}

Peu nombreuses sont les décisions dans lesquelles le juge administratif se réfère aux usages pour trancher un litige et départager les parties. Il reconnaît néanmoins une fonction à l'usage qu'il convient d'examiner (A). En appliquant les usages, il arrive que le juge signale leur normativité. Se pose alors la délicate question de savoir comment l'usage, simple fait, peut accéder à la qualité de coutume, règle de droit, en droit administratif camerounais $(\mathrm{B})$.

\section{A. La fonction incontestée de l'usage}

Les usages que le juge administratif applique dévoilent un double visage, relativement à leur fonction : ils sont un complément indispensable aux textes et parfois, ils prennent leur contre-pied. La position du juge administratif demeure vacillante sur ce dernier aspect.

\section{L'usage, complément indispensable de la réglementation}

La fonction de comblement des lacunes des textes est sans conteste le rôle le plus important attribué à l'usage dans la jurisprudence du juge administratif. Lorsqu'il s'appuie sur l'usage pour combler les lacunes de la réglementation, sa démarche se révèle suffisamment hardie $^{43}$. L'insuffisance des textes donne libre cours à l'usage, et parfois l'instruction ne

L'attitude de timidité et de prudence que le juge adopte lorsqu'il applique l'usage dans le jugement Société Commerciale Africaine et Office National de Commercialisation des Produits de Base (ONCPB) c/ Etat du Cameroun ( ${ }^{\circ} 1 / \mathrm{CS} / \mathrm{CA}$, du 29 oct. 1981), semble isolée. Sans se référer formellement à l'usage invoqué par la direction de l'ONCPB pour fonder l'accord donné par cette 
révèle pas toujours que l'usage est allégué par une partie. On a alors l'impression que le juge recherche d'office l'usage pour trancher le litige qui lui est soumis.

Dans le domaine particulier des pratiques administratives, le juge affirme que l'attitude constante de l'administration peut créer des droits au profit des administrés. La formation des usages est indifférente, seules comptent, leur reconnaissance par le juge et la fonction qu'il leur assigne. Qu'il découle clairement d'une série de précédents ${ }^{44}$ ou qu'il apparaisse à la suite de la clarification de l'attitude de l'administration par le juge, l'usage apporte une réponse au problème que les textes ne peuvent fournir.

Ainsi, un acte administratif peut découler de l'usage, sans être formellement élaboré par l'administration. Il faut citer l'important considérant de l'arrêt Dame Mackongo AgnèsFlore $^{45}$ qui révèle une pratique suivie dans l'affectation des fonctionnaires. Le juge administratif relève, «...que sans répondre à cette demande mutation (celle de Dame Mackongo), le ministre des affaires étrangères a préparé et procédé officiellement et sans réserve aucune, à la mise en route de cet agent pour New York; qu'une telle attitude ne peut constituer, au regard de la coutume administrative, qu'une autorisation d'absence non équivoque accordée par cette administration à son agent ».

Les termes que le juge administratif utilise pour identifier l'usage en révélant son rôle supplétif sont suffisamment évocateurs. C'est cette attitude constante que le juge signale, en l'absence d'une prescription formelle de la réglementation. On note par conséquent que le juge n'est pas attaché au formalisme ; bien au contraire, il invite à tenir compte, dans l'élaboration des actes administratifs, à la fois des actes écrits, mais aussi de ceux accomplis en vertu de la pratique. Tous sont des actes officiels et, comme tels, ils ont la même valeur au niveau des effets de droit qu'ils produisent ${ }^{46}$.

entreprise publique à la Société Commerciale Africaine afin de lui permettre de procéder à la vente du cacao à l'exportation, le juge administratif reconnaît la validité de cette transaction au motif que l'accord de l'ONCPB « ...n'est prescrit par aucun texte».

L'usage qui s'est constitué à la suite des précédents dans l'arrêt Ndjeudji Maurice (supra, note 31) a comblé les lacunes du statut de la magistrature en matière d'avancement et de promotion des magistrats. C'est ce rôle que lui reconnaît Paul Debelmas lorsqu'il relève que «l'insuffisance des textes est une cause importante de formation d'usages dont l'origine n'est bien souvent qu'un simple précédent mais qui sont cependant un complément utile et même nécessaire », in Les pratiques administratives comme source de droit, thèse, Toulouse, 1932, p. 52.

Op. cit., note 22.

L'autorisation d'absence « établie » ou justifiée par l'usage est un acte administratif à part entière. Il est pris par l'administration dans le but de modifier l'ordonnancement juridique, défini par $M$. Virally comme un "ensemble de droits et obligations existant à un moment donné dans une société donnée ». Lire La Pensée juridique, Paris, LGDJ, 1960, p.13, note 5. En outre, si l'on admet généralement, en droit administratif, que les actes gestuels d'une autorité administrative, 
Dans cet office le juge administratif est animé du double souci d'équité et d'égalité, quand il prend position en faveur des usages, dans le but de combler les insuffisances des textes. Sa position est empreinte d'équité, dans la mesure où il se libère des textes, du reste lacunaires ${ }^{47}$, pour faire parler sa conscience et réaliser la justice ${ }^{48}$. Il ne fait pas de doute, par ailleurs, que le juge, en admettant que l'administration puisse se lier par ses précédents ou par la pratique constante, entend assurer le respect du principe d'égalité. La «règle » établie par l'usage dans l'arrêt Ndjeudji Maurice étant d'admettre le cumul de promotion de grade et de franchissement d'échelon à la même date, le principe d'égalité aurait sans doute été violé si le juge n'avait pas accédé à la demande du requérant, alors que d'autres magistrats placés dans les mêmes conditions statutaires que lui avaient bénéficié du traitement qu'il revendiquait ${ }^{49}$.

Cette instruction est révélatrice du caractère singulier de la démarche du juge quand il se prononce sur des usages autres que les pratiques administratives. Le juge admet, parfois clairement, que l'usage s'applique en l'absence de texte. On peut citer l'exemple du jugement Ngoumou Richard ${ }^{50}$, dans lequel la Chambre Administrative juge que, «...quoiqu'un texte ne définit point explicitement le règlement des factures, il est de pratique que les services soient réglés par chèque libellé au nom du fournisseur ou en espèces... ».

Le rôle que le juge administratif assigne à l'usage praeter legem n'est plus à démontrer. Il justifie amplement la place de l'usage en droit administratif camerounais. Toutefois, la fonction que l'usage exerce à l'encontre de la loi ne saurait passer inaperçue.

par exemple, peuvent constituer des actes administratifs (lire G. Dupuis, M.-J. Guédon et $P$. Chrétien, Droit administratif, $7^{\text {ème }}$ édition, Paris, Dalloz 2000, p. 428-429), on ne voit pas pourquoi on refuserait cette qualité aux actes établis par la pratique, exprimant la même volonté de l'administration.

On note dans l'arrêt Ndjeudji Maurice précité que, c'est parce que l'art. 45(1) du statut de la magistrature (décret n ${ }^{\circ} 75 / 596$ du 23 août 1975, J.O. du $1^{\text {er }}$ sept. 1975, p. 1056) n'a pas pu fournir une réponse appropriée à la question du cumul de promotion de grade et de franchissement d'échelon à la même date, que l'usage a pu s'imposer. Ce texte dispose que «la promotion $d u$ deuxième grade au troisième grade comporte attribution pour compter de ladite promotion, de l'échelon de rémunération immédiatement supérieur à celui dont le magistrat promu bénéficiait au deuxième grade $»$.

$P$. Cintura note, à ce sujet, que «le droit qui n'est pas exercé selon la lettre de la loi, permet aussi de réaliser la justice, en évitant de faire acception de personne ». Lire, "L'usage et la conception de l'équité par le juge administratif », Revue Internationale de Droit Comparé, $\mathrm{n}^{\circ} 3$, 1972, p. 658.

L. Richer note, à ce propos, que «l'administration est obligée d'appliquer une solution semblable à deux administrés en situation semblable non pas en raison de cette similitude, mais en raison de l'existence d'une règle générale », in "Les revirements de l'administration », Dalloz, 1984, chr., p. 84, colonne droite.

CS/CA, n ${ }^{\circ} 17$ du 23 novembre 1989, Ngoumou Richard contre Etat du Cameroun. 


\section{L'hérésie de l'usage contra legem}

L'étude de l'usage contra legem ne peut manquer de susciter un intérêt en droit administratif camerounais. La position ambivalente du juge administratif à cet égard dévoile sans équivoque sa volonté de consacrer cet usage dans certaines de ses décisions. Ainsi, le juge administratif se montre hostile à l'usage contraire au droit écrit, tandis que, dans d'autres circonstances, il dévoile sa faveur pour ce type particulier d'usage.

La position d'hostilité du juge à l'égard de l'usage contra legem est clairement exprimée dans le jugement Sté Assureurs Conseils Franco-Africains ${ }^{51}$ rendue en matière d'usages des assurances. Le juge se dévoile nettement à cette occasion comme garant du respect de la légalité. Ainsi, pour faire obstacle aux prétentions du ministère des Finances se fondant sur les usages des assurances afin de justifier sa décision de retrait de l'agrément de courtier d'assurances à la société requérante, la Chambre Administrative juge qu' "affirmer sans ambages...que l'administration peut décider à l'encontre des principes de droit positif et écrit, revient purement et simplement à faire l'apologie de l'illégalité et de l'arbitraire ».

Cette attitude d'hostilité du juge semble générale. Sa décision est de ce fait pertinente et ne manque pas de réalisme. Par son rejet de l'usage abrogatoire, le juge assure la garantie et le respect de la règle de droit. Il contribue de ce fait à la lutte contre l'imprécision, l'arbitraire et l'insécurité juridique, dont l'usage et la règle coutumière constituent généralement l'expression.

La démarche du juge ne souffre par conséquent d'aucune contradiction; elle devrait, en toute logique, s'imposer : l'administration est soumise à la légalité ; elle ne saurait agir en marge de cette dernière. Il peut paraître dès lors absurde que le juge administratif approuve en même temps l'application de l'usage contraire à la réglementation.

Lorsque le juge administratif se prononce en faveur d'une pratique contraire au droit écrit, il admet, d'une part, l'applicabilité de l'usage, sans le solliciter dans la résolution du litige. D'autre part, il applique formellement l'usage concerné dans un litige. La première situation se vérifie dans le jugement Youmbi André ${ }^{52}$, dans lequel le juge administratif retient que «...la coutume administrative invoquée par le requérant ne s'applique qu'au

CS/CA, n ${ }^{\circ} 62$ du 25 septembre 1980.

52 CFJ/CAY, 23 mars 1971. La pratique alléguée par le requérant et reconnue par le juge administratif consiste à admettre la candidature des fonctionnaires aux divers concours internes, sans tenir compte de leur âge. C'est en cela que l'usage contredit les dispositions de l'art. 8 du décret n ${ }^{\circ} 68 / \mathrm{DF} / 463$ du 5 déc. 1968 portant statut particulier du corps des fonctionnaires de la Sûreté Nationale (J.O. n²4 du 15 déc. 1968, p. 2244). 
deuxième concours...uniquement réservé aux fonctionnaires ». L'application de cette pratique contraire à la réglementation aux situations antérieures, semble avérée, sinon le juge n'aurait pas révélé cette « coutume administrative».

On doit surtout observer une autre manifestation de l'usage contra legem, en citant un considérant dans lequel le juge applique une pratique «constante», contraire à la réglementation : «...qu'il est de pratique constante que le Premier ministre nomme par arrêté les cadres subalternes de la Sûreté nationale; qu'il est par conséquent habilité à procéder à leur licenciement; que la prétention d'Emougou que l'arrêté qui le licencie est illégal pour n'avoir pas été signé par le Président de la République est sans fondement ${ }^{53}$.

Les différentes manifestations de l'usage contra legem qui viennent d'être exposées ne peuvent manquer de susciter des interrogations. Il est permis, en effet, de se demander si l'usage contraire au droit écrit qui se manifeste «potentiellement » a, au regard du droit, la même valeur que celui que le juge sanctionne «effectivement». Cette préoccupation ne manque pas d'intérêt. On l'a vu, dans le jugement Youmbi André, le juge ne tranche pas le litige sur la base de l'usage invoqué par le requérant, puisqu'il ne l'applique pas. Si l'application effective de l'usage contraire au droit écrit ne fait pas de doute dans la jurisprudence du juge administratif $^{54}$, en revanche, son application potentielle ne peut manquer de soulever certaines difficultés.

Pour tenter de lever toute équivoque, il faudrait s'attarder sur la terminologie utilisée par le juge, en procédant à l'interprétation du terme «applique ». On peut, d'emblée, penser que pour que l'usage s'applique, il faut nécessairement que le juge le sollicite pour trancher le

CS/AP, 5 juillet 1973, Emougou Michel Gabriel, supra, note 18. La compréhension de ce considérant nécessite une clarification, au regard du rôle de l'usage dans l'issue du litige. La pratique « constante» de la nomination des cadres subalternes, contraire au droit écrit, est clairement établie. En effet, aux termes de l'article 4 du décret n ${ }^{\circ} 68 / \mathrm{DF} / 431$ du 29 octobre 1968 portant statut général de la Sûreté Nationale, «le Président de la République a le pouvoir de nomination et le pouvoir de gestion pour tous les grades et emplois. Il peut déléguer tout ou partie de chacun de ses pouvoirs, notamment aux Premiers ministres des Etats fédérés en ce qui concerne les personnels mis à leur disposition et les emplois relevant de leur compétence». Mais c'est le licenciement du requérant qui est contesté, et non sa nomination. Il serait donc hasardeux d'affirmer que le licenciement des fonctionnaires relève aussi de la «pratique constante ». Néanmoins, le licenciement des cadres subalternes de la police par le Premier ministre est lui aussi contraire à la réglementation, étant donné qu'il se fait en marge des prescriptions de cette dernière. Notons que s'il y avait eu délégation de pouvoirs, le juge se serait appuyé sur celle-ci, et non sur la pratique constante de l'administration.

Le juge administratif établit le lien entre les deux faits (fait de nomination et fait de licenciement), à travers la procédure du parallélisme des compétences : c'est la nomination par l'usage, qui habilite le Premier ministre à licencier par arrêté les personnels subalternes de la Sûreté Nationale. Supra, note 53. 
litige porté à sa connaissance. Dans ce cas, l'usage que le juge signale ne «s'applique » pas, lorsque son existence établie n'apporte pas une solution au litige, c'est-à-dire, que le juge ne le sanctionne pas. On peut aussi relever que l'usage s'applique lorsque le juge admet qu'il est susceptible de fournir une réponse au litige qui lui est soumis, sans nécessairement sanctionner l'usage en question ${ }^{55}$. Devant ces deux conceptions du terme «s'applique », quelle est celle qui devrait prévaloir?

On n'hésiterait pas à rejeter la deuxième conception, étant donné que la décision retenue par le juge administratif ne se fonde pas sur l'usage. L'usage ne produit pas des effets de droit, pourrait-on souligner, car le juge ne l'applique pas au litige. La candidature du sieur Youmbi est rejetée parce qu'il ne remplit pas les conditions d'âge requises. Toutefois, en relevant que la «coutume administrative invoquée par le requérant ne s'applique qu'au deuxième concours...uniquement réservé aux fonctionnaires », le juge administratif admet que l'usage puisse déroger au texte. Ce faisant, il reconnaît nécessairement l'applicabilité de l'usage contra legem dans l'ordre juridique. Concrètement, le juge voudrait dire que la prétention du requérant aurait sans doute prospéré, s'il avait invoqué l'usage en cause dans le cadre du concours qui était le sien, c'est-à-dire celui réservé aux fonctionnaires. Raisonner de cette façon ne semble pas incohérent.

Il ne paraît pas par conséquent excessif d'affirmer que l'usage contra legem est susceptible d'être sanctionné par le juge ; tout dépend du cadre où il est suivi ; celui invoqué par le requérant n'est pas approprié. Cette vision des choses ne semble pas erronée. Le juge ne procède pas à une simple identification de l'usage ; il entend attacher certains effets juridiques à la pratique concernée. Cette pratique prend de ce fait le contre-pied de la réglementation établie, et le juge lui reconnaît le rôle d'usage contra legem ${ }^{56}$.

Le problème théorique qui se pose ici se rapproche de l'effectivité du droit, qui, comme le note François Rangeon, "n'appartient pas à la définition de la règle de droit», même si elle «mesure les écarts entre le droit et son application». Lire ses « Réflexions sur l'effectivité du droit », in Les usages sociaux du droit, PUF, 1989, p. 126-127. Le raisonnement adopté pour prouver l'applicabilité de l'usage, alors même que le juge ne le sanctionne pas, s'adapte à la norme juridique en général. Comme le souligne G. Teboul, si la sanction constitue le critère de juridicité, «pour qu'une norme appartienne au monde du droit, il n'est pas nécessaire qu'elle soit effectivement sanctionnée. Ce qui compte, c'est qu'elle puisse faire l'objet d'une sanction, qu'il existe une sanction à titre potentiel ». («A propos de la coutume dans la jurisprudence administrative. Dialogue intérieur ». Mél. René Chapus, Paris, Montchrestien, 1992, p. 593). S'agissant plus particulièrement de la coutume, l'auteur relève que lorsqu'un usage est énoncé sous une forme normative dans les motifs d'une décision, on peut estimer que l'on se trouve en présence d'une règle coutumière, alors même que ledit usage - qui prescrit ou permet un comportement n'est pas sanctionné. Cette position a été adoptée par le Conseil d'Etat français dans l'arrêt C.E., 8 juillet 1927, Sieur Lecamus et autres, Rec. Lebon, p. 765. l'applicabilité de l'usage. Elle ne nuance pas non plus son propos pour dire, par exemple, que la 
L'attitude que le juge administratif camerounais adopte en faveur de l'usage contraire au droit écrit trouve difficilement une justification. En remarquant qu'il adopte uniquement cette démarche pour fonder la légalité de certaines pratiques administratives, il semble admettre que l'administration peut «défaire » un texte dont elle est à l'origine, par une pratique contraire. Par cette attitude, le juge fragilise son action, et les dangers d'une telle position sont réels pour la légalité et l'Etat de droit dont la protection incombe pourtant à l'autorité juridictionnelle.

Si applicabilité et application de l'usage contra legem ne se distinguent pas fondamentalement par leurs effets sur le droit, il reste qu'en droit administratif camerounais, c'est l'application de l'usage qui donne un sens décisif à l'issue d'un litige. On comprend pourquoi la question de la normativité de l'usage se vérifie uniquement dans le cadre de son application effective.

\section{B. Eléments de normativité de l'usage}

Le problème posé par le passage du fait au droit révèle toute la délicatesse du maniement de l'usage. Dans l'ensemble, le juge administratif camerounais applique l'usage comme une véritable règle de droit. Il lui revient de révéler qu'une règle de droit, c'est-à-dire la coutume, établie dans un groupe social est engendrée par l'usage. Cette règle préexiste au litige, et le juge se contente de révéler sa juridicité ${ }^{57}$.

coutume administrative «aurait pu s'appliquer »...Rien ne l'empêchait d'adopter cette formulation qui, en fait, n'aurait rien changé au fond du problème. Une règle qui «s'applique » ou qui «aurait pu s'appliquer», porte, dans tous les cas, les germes de son applicabilité, ce qui signifie que le juge reconnaît, en définitive, qu'elle est applicable.

La question de l'application ou de la création du droit par le juge continue d'alimenter la controverse. L'idée que le juge administratif applique la coutume sans la créer est contredite par le Professeur J. Chevallier. Pour l'auteur, la coutume ne préexiste pas à l'intervention du juge ; une règle de droit nouvelle peut seulement découler de cette intervention, étant donné que c'est la sanction juridique, celle de la violation de l'usage par le juge qui transforme le phénomène coutumier en règle jurisprudentielle. Cette observation est valable pour l'ensemble du phénomène coutumier (Lire «La coutume et le droit constitutionnel français », RDP 1970, nº, p.1405). Le danger d'une telle vision des choses est de faire du juge, organe non élu, un donneur de normativité, son activité créatrice allant au-delà de la simple interprétation des normes. Contre l'idée de création du droit par le juge, voir G. Teboul, «A propos de la coutume... », op. cit., p. 591-592; Usages et coutume..., p. 133-134. Cette dernière préoccupation semble partagée par M. Denys de Bechillon, lorsqu'il note le juge « signale à coup sûr que la juridisation a bien eu lieu, et que l'on se trouve en présence d'une norme patentée ». Lire «Qu'est-ce qu'une règle de droit ? », Paris, éd. Odile Jacob, 1997, p. 243. 
Le juge administratif signale implicitement l'existence d'une règle coutumière dans une affaire, lorsque tirant les conséquences de l'irrespect de l'usage, il sanctionne la violation de celui-ci par un membre du groupe social dans lequel l'usage tisse sa toile. Il révèle dans ce cas que l'usage se transforme en règle de droit. C'est dans cette optique qu'il faut comprendre la démarche que le juge adopte dans le jugement Ngoumou Richard ${ }^{58}$. Après avoir observé que «...quoiqu'un texte ne définit point explicitement le règlement des factures, il est de pratique que les services soient réglés par chèque au nom du fournisseur... », le juge considère comme constitutive d'une faute la violation de la pratique concernée lorsque, pour condamner le sieur Ngoumou, il décide, «que dès lors nous ne pouvons que conclure à une irrégularité de gestion commise par le requérant». Comme le révèle avec pertinence le Professeur Chevallier, la sanction de la violation de l'usage permet à celui-ci d'être «élevé sans discussion possible au rang de règle de droit " ${ }^{59}$, car l'intervention du juge fixe les sujets de droit sur le fait que leur comportement contraire à l'usage fera l'objet d'une sanction.

Le juge administratif révèle ainsi que l'usage prescrit un comportement. On observe cette attitude du juge lorsqu'il signale l'existence d'une « règle » établie par la pratique dans la promotion des magistrats. En annulant un acte administratif méconnaissant la pratique établie, le juge impose à l'administration le respect de l'usage concerné. Ce faisant, il reconnaît sa normativité ${ }^{60}$. La légalité de cet usage est clairement révélée par le juge lorsque, sanctionnant la violation de la pratique, il admet que l'administration a commis une illégalité ${ }^{61}$.

Il arrive aussi que l'usage, établi sans contestation aucune, confère le droit à l'autorité administrative d'adopter un certain comportement. C'est ce qui est observé lorsque l'administration procède à l'affectation de ses agents sans obligation de sa part de répondre

59 J. Chevallier, «La coutume et le droit constitutionnel français », supra, p. 1402-1403.

Supra, note 31 . C'est la « règle » établie dans la promotion des magistrats dans cette administration, et clairement révélée par le juge dans cette affaire qui a amené ce dernier à annuler le décret litigieux qui attribuait au requérant un indice de rémunération inférieur à celui de ses collègues placés dans les mêmes conditions statutaires que lui, et ayant bénéficié de la pratique établie.

Dans l'arrêt Ndjeudji Maurice, l'Assemblée Plénière relève que «considérant que si l'illégalité ainsi commise suffit à engager la responsabilité de l'Etat... ». L'administration a donc commis une illégalité parce qu'elle a violé la « règle » du cumul d'avancement d'échelon et de promotion de grade à la même date, établie par l'usage, dans la promotion des magistrats. La normativité de l'usage est renforcée par le fait que le juge admet que sa violation peut engager la responsabilité de l'Etat. 
aux demandes de mutations qui lui sont adressées ${ }^{62}$. En confirmant la régularité d'une telle affectation, et en annulant la décision du secrétaire d'Etat à la Fonction publique constatant l'absence irrégulière de la requérante, le juge administratif admet que le ministre des Affaires étrangères a le droit d'affecter les agents de son administration conformément à la coutume administrative établie ${ }^{63}$. Il signale de ce fait une règle coutumière, issue de l'usage.

Par ailleurs, le juge administratif révèle que l'usage habilite les chefs traditionnels à se faire représenter par leurs «capitas » (remplaçants ou adjoints) à la cérémonie de désignation d'un chef supérieur, alors même que la réglementation est muette sur la question ${ }^{64}$. Les termes sur lesquels le juge s'appuie sont évocateurs d'une certaine normativité. Ainsi, lorsque le juge prescrit une enquête dans le but de savoir si la « «coutume admet» la représentation des chefs traditionnels à la cérémonie de désignation d'un nouveau chef ${ }^{65}$, et que, vidant son avant dire droit, il décide qu'un chef peut (en vertu de l'usage) ${ }^{66}$ se faire représenter, il faut reconnaître que c'est l'usage qui «admet », certes implicitement, ladite représentation, en l'absence d'un texte régissant cette matière.

On ne peut pas dire que l'usage engendre le droit à travers la seule terminologie utilisée par le juge. Mais il ne fait pas de doute que le langage du juge participe à la révélation de la normativité de l'usage. En effet, c'est parce qu'il est avéré que l'usage (désigné « coutume ») admet la représentation des chefs traditionnels que le juge administratif trouve une issue à l'affaire Menyé. Aussi, à la question de savoir si un chef traditionnel de niveau

CFJ/CAY, arrêt n²01 du 18 août 1972, Dame Mackongo Agnès-Flore. Il est avéré que les textes applicables au moment de l'affaire, notamment, l'ordonnance $n^{\circ}$ 59-70 du 27 nov. 1959 fixant le statut des fonctionnaires de l'Etat du Cameroun et le décret-loi $\mathrm{n}^{\circ}$ 63-1 du 9 janv. 1963, n'entraient pas dans les détails de gestion des fonctionnaires, et par conséquent ne faisaient obligation aux ministres de répondre aux demandes d'affectation des agents de leurs administrations.

S'il y avait un texte empêchant l'administration de procéder de la sorte, i.e. lui imposant l'obligation de répondre aux demandes de mutation de ses agents, et que le juge approuvait la démarche contraire de l'administration, on aurait été confronté à une coutume contra legem. Ce qui n'est pas le cas.

Il faut distinguer cette situation de celle où l'usage est «confirmé par la loi » que nous avons écartée de cette étude (supra, note 9). Le problème de la désignation des chefs traditionnels observé de part et d'autre se pose dans deux contextes différents. 
inférieur peut ou a le droit de se faire représenter à la cérémonie de désignation d'un autre chef de niveau supérieur ${ }^{67}$ dans le groupe social concerné, on répondrait par l'affirmative.

Dans tous les cas cités, l'usage comble les lacunes de la réglementation. Comme le relève M. Denys de Béchillon, c'est en tant que règle juridique qu'il subit la sanction du juge ${ }^{68}$. A ce titre, la coutume issue de l'usage se positionne comme une règle opposable à tous. C'est dans son rôle supplétif que l'usage se révèle comme une nécessité juridique. En «normant » les comportements des sujets de droit, il lui arrive de jouer le rôle d'une véritable règle coutumière, c'est-à-dire d'une règle de droit. Il ne relève donc pas de l'utopie de parler de coutume praeter legem en droit administratif camerounais, sa place dans ce droit étant suffisamment justifiée.

\section{Conclusion}

L'usage trouve une place dans la jurisprudence du juge administratif camerounais, à côté du droit écrit. Son rôle est, de ce fait, difficilement contestable. Il serait cependant irréaliste de prétendre lui accorder une importance considérable dans la jurisprudence de l'ordre administratif. Son importance par rapport au droit écrit est certes limitée, mais son rôle effectif ne semble pas faire de doute.

C'est surtout lorsque l'usage donne naissance à une règle de droit révélée par le juge, que son étude et son observation prennent un relief particulier en droit administratif camerounais. La coutume qui en découle est valide ${ }^{69}$, dès lors que le juge administratif l'applique pour dire le droit dans plusieurs litiges. Elle devrait par conséquent être reconnue en droit administratif comme une composante à part entière de l'ordre juridique interne qui, jusqu'ici, réservait uniquement une place à la coutume en droit privé.

L'art. $1^{\text {er }}$ du décret $\mathrm{n}^{\circ} 244$ du 04 février 1933 fixant le statut des chefs indigènes prévoit que La hiérarchie des chefs comprend : les chefs supérieurs ; éventuellement des chefs de groupement; et des chefs de village.

«Les chefs indigènes sont choisis autant que possible au sein des familles appelées à exercer héréditairement le commandement, après consultation, pour les chefs de région et de groupement, des chefs de village intéressés, et ces derniers, des chefs de famille composant le village ».

Qu'est-ce qu'une règle de droit ?, supra, p. 241.

69

Kelsen définit la validité comme « le mode d'existence spécifique des normes », Théorie pure du droit, préc., note 14, p. 18. 